\title{
Output Feedback Sensitivity Functions Under Signal to Noise Ratio Constraint.
}

\author{
A.J. Rojas ${ }^{\dagger}$, J.H. Braslavsky and R.H. Middleton
}

\begin{abstract}
We analyse the control output feedback sensitivity function which results from imposing the minimal Signal-to-Noise Ratio requirement needed for stabilisability. The Signal to Noise Ratio constraint used here includes the effect of a band limited, additive coloured Gaussian noise communication link and a (non) minimum phase unstable continuous (discrete) linear time invariant (LTI) plant with time delay.

Target performance is introduced in a form that directly implies an additional term in the required SNR compared to the case of stabilisability only.

In a first approach performance is sought as output disturbance rejection giving guaranteed reference tracking at zero frequency (integral action) and compensation for perturbations over a range of frequencies. It is noted that the resulting additional cost can be made vanishingly small by making the closed loop arbitrarily slow.

In a second approach we define frequency bounds for the magnitude of the output sensitivity function. These bounds will guarantee low error reference tracking and rejection of disturbances over a non trivial range of frequencies. Based on this, a lower bound for the required extra SNR that satisfies the mentioned sensitivity magnitude bounds is proposed and its tightness discussed.
\end{abstract}

\section{INTRODUCTION.}

Control over networks has become an area of growing interest in recent years with works such as [1], [2], [3], [4], [5] and [6]. See also [7] and the references therein.

Generally, the communication link involves some preand post-processing of the signals that are sent through a communication channel, for example, filtering, analogto-digital (A-D) conversion, coding, modulation, decoding, demodulation and digital-to-analog (D-A) conversion.

Of the two possible configurations for the location of the idealised communication channel (measurement path and control path), we consider the case of a communication channel over the control link. Such a setting is common in practice and arises, for example, when actuators are far from the controller and have to communicate through a (perhaps partially wireless) communication network.

$\dagger$ : corresponding author.

This work was supported by The ARC Centre for Complex Dynamic Systems and Control, 2308, Australia.

A.J. Rojas is with the School of Electrical Engineering and Computer Science, The University of Newcastle, 2308, Australia alejandro.rojas@newcastle.edu.au.

J.H. Braslavsky is with the School of Electrical Engineering and Computer Science, The University of Newcastle, 2308, Australia julio.braslavsky@newcastle.edu.au.

R.H. Middleton is with the School of Electrical Engineering and Computer Science, The University of Newcastle, 2308, Australia richard.middleton@newcastle.edu.au.
Nonetheless, in a Single-input Single-output (SISO) linear time invariant (LTI) setting, both forms are equivalent, and it is a simple matter to restate the results for the case of where measurement is performed over a communication channel.

Stabilisability of the resulting feedback loop has been studied in relation to quantisation, bit rate limitations, bandwidth constraints and time delays over the communication channel. A line of investigation using topological and entropy concepts is pursued in [8], [9] and [10]. More recently in [11] interesting results have been presented on generalisations of Bode's Integral Theorem, including limits implied by the communication channel capacity.

Stabilisability in terms of Signal to Noise ratio has been primarily studied in [12], [13], [14], [15], and [16]. The analysis there includes the effects of plant non-minimum phase zeros and time delays, both in the continuous and discrete time setting, in an output feedback and state-space feedback scheme, whilst the communication link has been studied for the case of infinite bandwidth, band limited, white or coloured additive Gaussian noise.

The present article takes inspiration from results in [16], which model the communication channel through the idealisation of a band limited, additive coloured Gaussian noise (ACGN) channel, imposing a power constraint on the channel input signal. Thus the stabilisability problem is expressed through a bound on the signal to noise ratio (SNR) defined by the imposed power constraint and the white noise power spectral density.

In the present paper, as in [16] before, we neglect all preand post- signal processing involved in the communication link, which is then reduced to the communication channel itself. The bandwidth constraint on the communication channel may be imposed, for example, to avoid interference between different channels in a communication system, whilst the coloured noise may be imposed as a more realistic feature for a communication channel than the white noise case studied in [12], and [13].

In control theory important feedback loop properties are defined by the sensitivity function and/or the complementary sensitivity function, see for example [17]. A common design requirement, that can be quantified through the sensitivity function, is guaranteed reference tracking and simultaneous compensation for possible modelling errors (or disturbances) at zero frequency. In order to achieve the above design requirement the presence of an integrator in the controller is required. In what follows the design requirement at zero frequency and the presence of an inte- 
grator in the controller will be considered interchangeable concepts, which we will call in short integral action.

The main result of this work is an expression for the sensitivity function of an output feedback loop when the minimal SNR required to guarantee stabilisability is imposed together with a band limited ACGN communication channel both in the continuous and discrete framework. Also a lower bound for the extra SNR requirement for almost achieving disturbance rejection (i.e., $|T(j \omega)| \approx 1$ and $T$ the complementary sensitivity) over a given bandwidth defined by the user is presented.

The paper is organised as follows: in Section 2 we address the resulting sensitivity function for the continuous (discrete) time output feedback stabilisability problem over a power constrained, bandwidth limited ACGN channel. Section 3 introduces a lower bound for the extra SNR needed to guarantee stabilisability and disturbance rejection over a given range of frequencies. Finally Section 4 presents concluding remarks. Due to the page constraint most proofs are referred to the Appendix of [18] (under review) and can be provided upon request to the corresponding author.

\section{Sensitivity for the Minimal SNR With GUARANTEED STABILISABILITY.}

Consider the case of a plant with pure time delay $\tau, m$ different unstable poles and $q$ different NMP zeros.

$$
G=G_{o} e^{-s \tau}=\frac{B_{z G} N_{o}}{B_{p} M_{o}} e^{-s \tau},
$$

where $N_{o}$ and $M_{o}$ are stable proper, minimum phase transfer functions and

$$
B_{p}=\prod_{i=1}^{m} \frac{s-p_{i}}{s+\bar{p}_{i}}, \quad B_{z G}=\prod_{j=1}^{q} \frac{s-z_{j}}{s+\bar{z}_{j}} .
$$

Also consider the bandwidth limited ACGN channel, depicted in Figure 1, where filter $F(s)$ is stable, biproper and models the bandwidth limitation. Filter $H(s)$ is also stable, biproper and colours the zero-mean white Gaussian noise $w(t)$. In particular we assume that there are $f$ NMP zeros located at filter $F(s)$, that is $F=B_{z F} \tilde{F}$. In general if a precise distinction about the origin of any given NMP zero is not required we will use the notation $B_{z}=B_{z G} \cdot B_{z F}$.

The main result from [16] is that the SNR required for stabilisability satisfies

$$
\frac{\mathcal{P}}{\Phi}>\sum_{i=1}^{m} \sum_{j=1}^{m} \frac{r_{i} \bar{r}_{j}}{p_{i}+\bar{p}_{j}} e^{\left(p_{i}+\bar{p}_{j}\right) \tau}
$$

where each $r_{i}$, with $i=1, \cdots, m$ is given by

$$
r_{i}=2 \operatorname{Re}\left\{p_{i}\right\} B_{z}^{-1}\left(p_{i}\right) \tilde{F}^{-1}\left(p_{i}\right) H\left(p_{i}\right) \prod_{\substack{k=1 \\ k \neq i}}^{m} \frac{p_{i}+\bar{p}_{k}}{p_{i}-p_{k}},
$$

where $\mathcal{P}$ is the channel input power constraint and $\Phi$ is the noise $w(t)$ power spectral density, as in Figure 1. Achieving the above result however implies a specific selection for

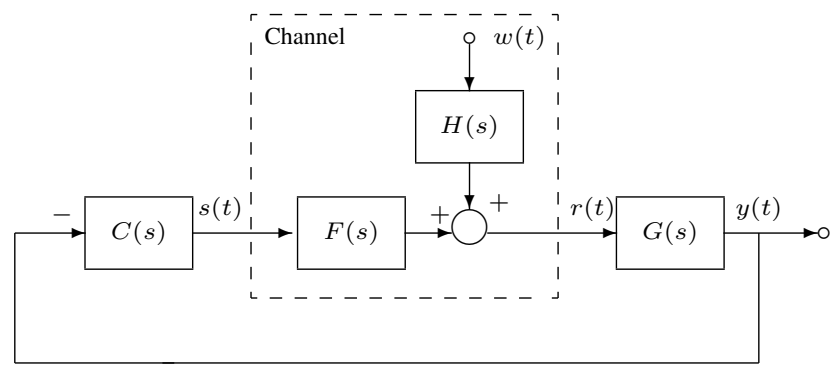

Fig. 1. Stabilisation via output feedback over a band limited ACGN channel.

the controller and through that, a specific structure for the sensitivity and complementary sensitivity of the output feedback loop. The expression for the sensitivity is explicitly given in the following theorem, whilst the expression for the complementary sensitivity can be deduced from it.

Theorem 1: Consider a continuous plant with $m$ different unstable poles $\left(p_{i}, i=1, \cdots, m\right), q+f$ different NMP zeros $\left(z_{j}, j=1, \cdots, q+f\right)$ (either from the plant or the channel) and time delay $\tau \neq 0$. The stabilising proper controller ${ }^{1}$ which achieves the minimal SNR required for stabilisability of the loop, in the presence of a band limited ACGN channel, will produce the following sensitivity for the output feedback closed loop

$$
\begin{aligned}
& \hat{S}(s)=1-e^{-s \tau} B_{z}(s) \tilde{F}(s) H^{-1}(s) . \\
& \cdot \sum_{i=1}^{m}\left(\frac{r_{i} e^{p_{i} \tau}}{s+\bar{p}_{i}} \prod_{\substack{j=1 \\
j \neq i}}^{m} \frac{s-p_{j}}{s+\bar{p}_{j}}\right),
\end{aligned}
$$

where $B_{p}$ and $B_{z}$ denote the Blaschke products for the unstable poles (from the plant) and NMP zeros (from the plant and/or channel model) defined as in (2).

Proof: see [18, $\S$ A.3.1].

The result above let us perceive more clearly what kind of behaviour we should expect from such closed feedback loop. For that take into consideration the following example.

Example 1: Take a plant with one real unstable pole $p$, one NMP zero $z$, no time delay (i.e. $\tau=0$ ) and a minimum phase bandwidth limited ACGN channel. The resulting complementary sensitivity, obtained from achieving the minimal possible SNR for stabilisability is given by

$$
T(s)=\frac{r}{s+p}\left(\frac{s-z}{s+z}\right) \tilde{F}(s) H^{-1}(s),
$$

where $r$ is given by

$$
r=2 \operatorname{Re}\{p\} B_{z}^{-1}(p) \tilde{F}^{-1}(p) H(p) .
$$

From (6) is possible to see clearly that the bandwidth of the obtained complementary sensitivity will be dictated by

\footnotetext{
${ }^{1}$ The expression for the infimal continuous-time sensitivity function, when the controller that achieves the SNR lower bound is improper, can be found in [18, p.63]. Here the infimal result is omitted in order to avoid details that would unnecessarily clutter the present discussion.
} 
$F$ or $H^{-1}$ only if their bandwidth is less than $p$, otherwise it will be constrained by the unstable pole to have a cut off frequency of $p[\mathrm{rad} / \mathrm{s}]$.

Next, the counterpart of Theorem 1 for the discrete output feedback loop case is given, (see also [16]).

Theorem 2: Assume a discrete plant with $m$ different unstable poles $\left(\rho_{i}, i=1, \cdots, m\right), q+f$ different NMP zeros $\left(\zeta_{j}, j=1, \cdots q+f\right)$, either from the plant or the channel model, and possible relative degree, $r$, greater than one. The stabilising controller which achieves the minimal SNR required for stabilisability of the loop, in the presence of a power constrained bandwidth limited ACGN channel, will impose the following sensitivity for the output feedback closed loop

$$
\begin{aligned}
& \hat{S}(z)=1+B_{\zeta}(z) B_{\rho}(z) \tilde{F}(z) H^{-1}(z) \sum_{k=1}^{r-1} \mu_{k} z^{-k} \\
& -B_{\zeta}(z) \tilde{F}(z) H^{-1}(z) \sum_{k=1}^{m}\left(\frac{r_{k}}{1-z \bar{\rho}_{k}} \prod_{\substack{j=1 \\
j \neq k}}^{m} \frac{z-\rho_{j}}{1-z \bar{\rho}_{j}}\right),
\end{aligned}
$$

where $B_{\rho}$ and $B_{\zeta}$ denote the discrete time Blaschke products for the unstable poles and NMP zeros

$$
B_{\rho}=\prod_{i=1}^{m} \frac{z-\rho_{i}}{1-z \bar{\rho}_{i}}, \quad B_{\zeta}=\prod_{j=1}^{q+f} \frac{z-\zeta_{j}}{1-z \bar{\zeta}_{j}},
$$

and

$$
\begin{aligned}
& r_{i}= \\
& \left(1-\left|\rho_{i}\right|^{2}\right)\left(\prod_{\substack{j=1 \\
j \neq i}}^{m} \frac{1-\rho_{i} \bar{\rho}_{j}}{\rho_{i}-\rho_{j}}\right) B_{\zeta}^{-1}\left(\rho_{i}\right) \tilde{F}^{-1}\left(\rho_{i}\right) H\left(\rho_{i}\right) \\
& \mu_{k}=\sum_{i=1}^{m} r_{i} \rho_{i}^{k-1} .
\end{aligned}
$$

Proof: see [18, § A.4.1].

Consider now the case where the required control design objectives have been successfully combined into a required target sensitivity for the continuous time output feedback loop. In this case, assuming that the target sensitivity differs from the one in (5), there will be an additional demand on the SNR to achieve the extra performance requirement. Note that different $S_{\text {per }}$ must satisfy the same interpolation constraints as $\hat{S}\left(S_{\text {per }}\left(p_{i}\right)=0 \forall i=1, \cdots, m\right.$ and $\left.S_{\text {per }}\left(z_{j}\right)=1 \forall j=1, \cdots, q\right)$.

The next theorem specifies the additional SNR created by the presence of $S_{\text {per }}$ in the continuous case.

Theorem 3: Consider a continuous time plant with $m$ different unstable poles, $q$ different NMP zeros, time delay $\tau$ and $f$ channel NMP zeros. Consider also a given target closed loop sensitivity, $S_{\text {per }}$. The SNR required to achieve simultaneously stabilisability and the required performance can be quantified as

$$
\frac{\mathcal{P}}{\Phi}>\sum_{i=1}^{m} \sum_{j=1}^{m} \frac{r_{i} \bar{r}_{j}}{p_{i}+\bar{p}_{j}} e^{\left(p_{i}+\bar{p}_{j}\right) \tau}+\left\|\left(S_{\text {per }}-\hat{S}\right) \tilde{F}^{-1} H\right\|_{\mathcal{H}_{2}}^{2},
$$

where $\mathcal{P}$ is the channel input power constraint and $\Phi$ is the noise $w(t)$ power spectral density. In (11) which $\sum_{i=1}^{m} \sum_{j=1}^{m} \frac{r_{i} \bar{r}_{j}}{p_{i}+\bar{p}_{j}} e^{\left(p_{i}+\bar{p}_{j}\right) \tau}$ takes into account the stabilisability requirement, whilst the expression $\left\|\left(S_{\text {per }}-\hat{S}\right) \tilde{F}^{-1} H\right\|_{\mathcal{H}_{2}}^{2}$ weights the performance requirement in terms of SNR.

Proof: see [18, $\S$ A.3.2].

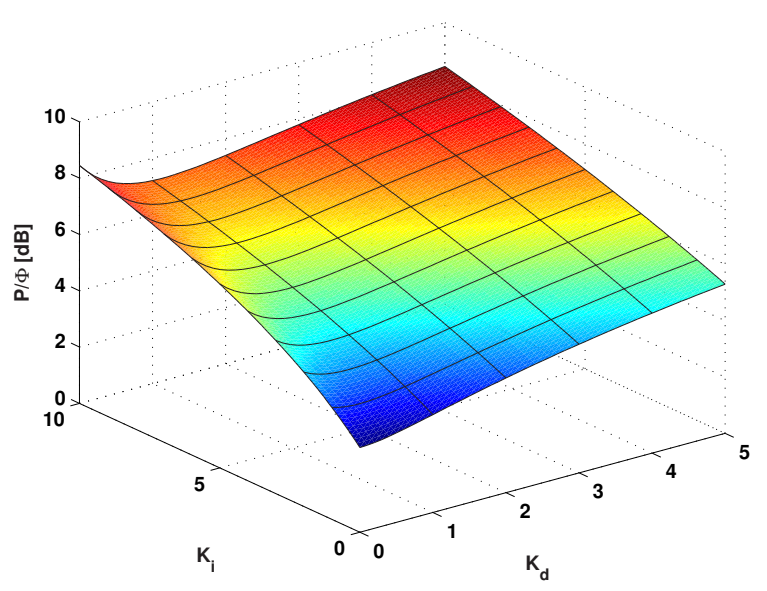

Fig. 2. SNR requirement as function of two PID controller parameters.

To better appreciate the relevance of the result expressed by Theorem 3 we start by the following example involving PID tuning.

Example 2: Consider the case of an AWGN communication channel with infinite bandwidth, and as an example a continuous time plant model given by

$$
G(s)=\frac{1}{s-1} .
$$

Theorem 1 defines the sensitivity function related to the minimal SNR required for stabilisability of the closed loop to be

$$
\hat{S}(s)=\frac{s-1}{s+1} .
$$

The complementary sensitivity is readily obtained as $1-$ $\hat{S}(s)$

$$
\hat{T}(s)=\frac{2}{s+1} .
$$

It is not hard to find out that the controller that achieves the above complementary sensitivity is a proportional controller set to 2. Consider now that the controller is changed to a PID parallel structure [19, p. 160]

$$
C_{P I D}=2+\frac{K_{i}}{s}+\frac{K_{d} s}{0.1 K_{d} s+1},
$$


with $K_{i} \in \mathbb{R}^{+}$and $K_{d} \in \mathbb{R}^{+}$, such as the controller is stabilising. The choice of a PID controller will not be optimal implying that some extra SNR is required, as predicted by Theorem 3. Figure 2 reports the extra SNR as a function of the parameters $K_{i}$ and $K_{d}$.

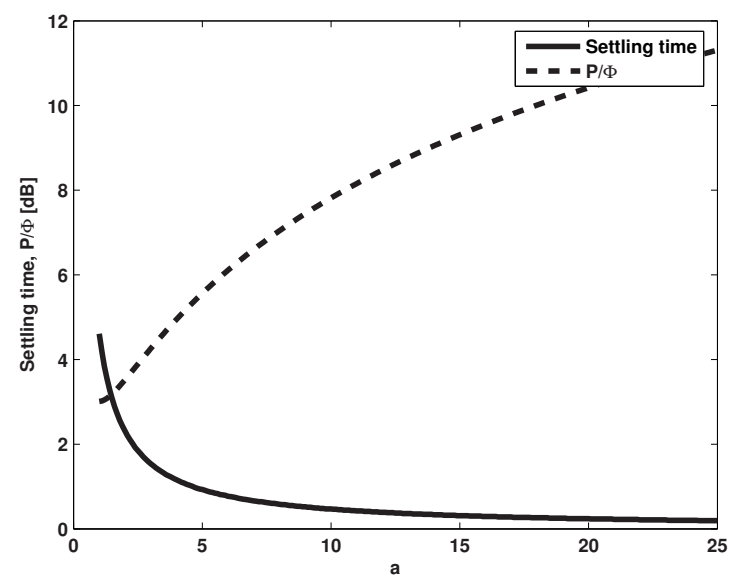

Fig. 3. Settling time, solid line, and SNR, dashed line, as functions of parameter $a$.

The example just presented reports the effect of a non optimal choice for the controller (specifically the case of a PID controller), but it does not explicitly address performance requirements. The following example touches the issue of performance directly.

Example 3: Consider the same plant and channel selection as in Example 2. The step response of the complementary sensitivity in (14) produces a settling value of 2 with a settling time of approximately 4.4 time units (here defined as the time in which the response of the system reaches $99 \%$ of the settling value). Define the error in settling value as the difference between the settling value of the output and the input. For the case of the complementary sensitivity in (14), since the input is a step of magnitude one and its settling value is 2 , the error in settling value amounts precisely to 1 and quantifies the reference tracking error at steady state.

Consider now that the user is not satisfied with such meager bandwidth for the closed loop and decides that it requires the following complementary sensitivity to be in place

$$
T_{p e r}(s)=\frac{1+a}{s+a},
$$

with $a$ positive real number and $a \geq 1$. Notice that $a$ can not be chosen to be less than 1 due to the optimal nature of the stabilising solution obtained by the minimal energy controller and its property of mirroring the unstable poles of the plant.

Theorem 3 tells us that this choice will force an increase in the lower bound for the SNR necessary to ensure stabilisability.

The unit step response for the complementary sensitivity defined in (16) has been tested for values of $a$ in a range between 1 and 25. Results are reported in Figure 3 and 4. It is possible to observe from Figure 3 how the closed loop settling time is reduced if the SNR is increased above the value needed only for stabilisability. Similarly in Figure 4 we can see that as the allowed SNR increases, the error in settling value for this example approaches 0 , which implies that disturbance rejection and successful reference tracking are taking place.

To better quantify these ideas take the case $a=5$ in which the SNR increases by the amount

$$
\left\|T_{\text {per }}-\hat{T}\right\|_{\mathcal{H}_{2}}^{2}=\left\|\frac{4(s-1)}{(s+1)(s+5)}\right\|_{\mathcal{H}_{2}}^{2}=1.6 .
$$

The settling time in this case, with the same $1 \%$ tolerance, is approximately 0.94 time units. Note that the base SNR, required for stabilisation, is 2 . So actually, if we can allow $80 \%$ more SNR, we can speed up the response by almost 5 times. The error in settling value for the same selection of $a$ amounts to 0.2 . The result in (17) quantifies how much the

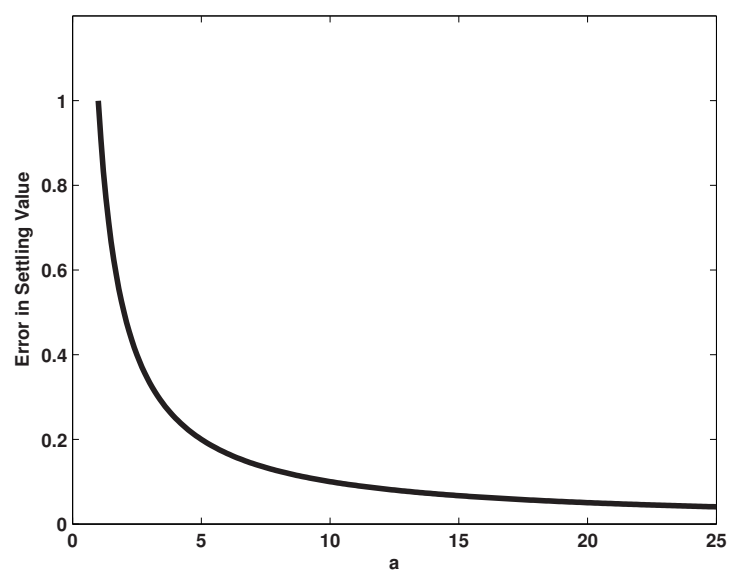

Fig. 4. Error in settling value as a function of parameter $a$.

SNR required for stabilisability will have to increase due to the target performance requirement.

\section{Sensitivity Reduction OVER A Given BANDWIDTH.}

A common desirable requirement when designing a control feedback loop is to ensure the presence of integral action in the loop, more precisely in the controller. This can be perceived as a performance requirement, but integral action by itself does not directly imply an additional SNR as the following example shows.

Example 4: Consider the case of an AWGN communication channel with infinite bandwidth. Also take the following transfer function as the plant model

$$
G(s)=\frac{1}{s-1} .
$$


It can be seen that the Youla parameter $Q(s)$ that guarantees integral action and satisfies the interpolation condition dictated by the unstable pole of the plant is

$$
Q(s)=(a+2) \frac{s+1}{s+a},
$$

with $a \in \mathbb{R}^{+}$. The complete result accordingly to Theorem 3 is given by

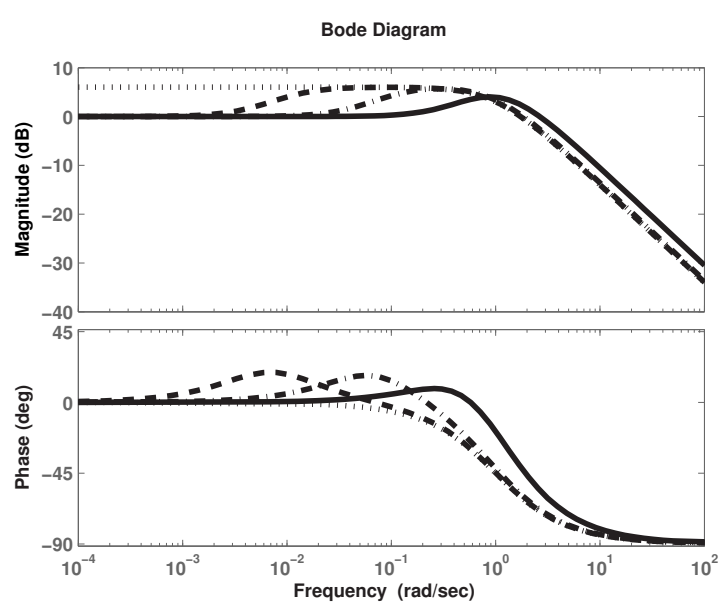

Fig. 5. Complementary sensitivities for $a=1$, solid line, $a=0.1$, dash-dotted line, $a=0.01$, dashed line and $a=0$, dotted line.

$$
\frac{\mathcal{P}}{\Phi}>2+\left(\frac{a}{2}\right)^{2}\left\|\frac{2}{s+a}\right\|_{\mathcal{H}_{2}}^{2}=2+\frac{a}{2} .
$$

If we consider $a \rightarrow 0$ we will regain the minimum result for assured stabilisability, but we will lose the disturbance rejection characteristic. This is more clearly appreciated through the complementary sensitivity expression as a function of $a$

$$
T(s)=\frac{(a+2) s+a}{(s+1)(s+a)} \stackrel{a \rightarrow 0}{\Rightarrow} \frac{2 s}{(s+1) s}=\frac{2}{s+1} .
$$

In Figure 5 it is possible to see the magnitude and phase Bode diagrams for different complementary sensitivities, with $a=1$, solid line, $a=0.1$, dash-dotted line and $a=0.01$ dashed line. The less we are willing to increase the SNR requirement the smaller the frequency range in which disturbances will be successfully rejected $(|T| \approx 1)$. From the example just presented, we have confirmation of [12] in which it is shown that the extra cost in terms of SNR required for integral action can be made arbitrarily small. Nonetheless the fact that the required SNR can be made arbitrarily small it is not of much practical use to us, since the associated bandwidth in which disturbance rejection is taking place also tends to zero. Another approach is to combine disturbance rejection with a bandwidth requirement by which it will have a real impact on the required SNR. To quantify this SNR calls for an upper bound, $S_{\max }$, in the sensitivity function to model the given bandwidth in which disturbance rejection, in terms of sensitivity reduction, is almost guaranteed.
The next theorem creates a lower bound for (11).

Theorem 4: Assume that the performance requirement of sensitivity reduction over a non trivial bandwidth is defined by a function $S_{\max }$, and that for any $S_{p e r}$ we have $\left|S_{\text {per }}\right| \leq\left|S_{\max }\right|$. Assume also that the complementary sensitivities in both cases are strictly proper, and therefore at high frequencies both magnitudes, $\left|S_{\max }\right|$ and $\left|S_{\text {per }}\right|$, will tend to one. Given the above the following holds true

$$
\begin{aligned}
&\left\|\left(S_{\text {per }}-\hat{S}\right) \tilde{F}^{-1} H\right\|_{\mathcal{H}_{2}}^{2} \geq \\
& \frac{1}{2 \pi} \int_{-\infty}^{\infty}\left[\left(\left|S_{\max }(j \omega)\right|-|\hat{S}(j \omega)|\right)^{2}\right. \\
&\left.\left|\tilde{F}^{-1}(j \omega) H(j \omega)\right|^{2}\right] d \omega .
\end{aligned}
$$

Proof: Take the extra term as defined in (11)

$$
\begin{aligned}
& \left\|\left(S_{\text {per }}-\hat{S}\right) \tilde{F}^{-1} H\right\|_{\mathcal{H}_{2}}^{2} \geq \\
& \frac{1}{2 \pi} \int_{-\infty}^{\infty}\left|S_{p e r}(j \omega)-\hat{S}(j \omega)\right|^{2}\left|\tilde{F}^{-1}(j \omega) H(j \omega)\right|^{2} d \omega \geq \\
& \frac{1}{2 \pi} \int_{-\infty}^{\infty}\left(\left|S_{p e r}(j \omega)\right|-|\hat{S}(j \omega)|\right)^{2}\left|\tilde{F}^{-1}(j \omega) H(j \omega)\right|^{2} d \omega
\end{aligned}
$$

From the condition of $\left|S_{\text {per }}\right| \leq\left|S_{\max }\right|$ we have

$$
\left|S_{\text {max }}\right| \geq\left|S_{\text {per }}\right| \Rightarrow\left(\left|S_{\max }\right|-|\hat{S}|\right)^{2} \leq\left(\left|S_{\text {per }}\right|-|\hat{S}|\right)^{2} \text {. }
$$

Replacing this inequality in (23) we obtain (22). Note that the strictly proper condition for the complementary sensitivities is needed to guarantee the convergence of (22).

In order to investigate how tight this lower bound result is, an example is investigated.

Example 5: For this example consider a plant with $m$ distinct unstable poles, with no zeros in the RHS nor time delay. The communication channel is AWGN (i.e. $F=\tilde{F}=$ 1 and $H=1$ ). The sensitivity function obtained by the related minimum energy problem is given by

$$
\hat{S}(s)=B_{p}(s)=\prod_{i=1}^{m} \frac{s-p_{i}}{s+\bar{p}_{i}} .
$$

Take also in account the case of a performance requirement defined through $\left|S_{\max }\right|$ as

$$
\left|S_{\text {max }}\right|=\left\{\begin{array}{cc}
\omega / \omega_{o} & 0 \leq \omega \leq \omega_{o} \\
1 & \omega_{o}<\omega .
\end{array}\right.
$$

By this choice, the lower bound in (22) can be obtained as

$$
\left\|S_{\text {per }}-\hat{S}\right\|_{\mathcal{H}_{2}}^{2} \geq \frac{\omega_{o}}{3 \pi} \text {. }
$$


To investigate how tight this bound is take the case of a choice of $S_{p e r}$ as

$$
S_{p e r}=\frac{s}{s+\omega_{o}} B_{p}(s) .
$$

The magnitude of this selection for $S_{\text {per }}$ is given by

$$
\left|S_{p e r}\right|=\frac{\omega}{\sqrt{\omega^{2}+\omega_{o}^{2}}} \leq \min \left\{1, \frac{\omega}{\omega_{o}}\right\} .
$$

Since the magnitude of $S_{\text {per }}$ is below the magnitude of $S_{\max }$, the bound is valid, but in this case we can also obtain the exact value of $\left\|S_{p e r}-\hat{S}\right\|_{\mathcal{H}_{2}}^{2}$

$$
\left\|S_{\text {per }}-\hat{S}\right\|_{\mathcal{H}_{2}}^{2}=\frac{1}{2 \pi} \int_{-\infty}^{\infty}\left|S_{\text {per }}-\hat{S}\right|^{2} d \omega=\frac{\omega_{o}}{2} .
$$

The result in (30) tells us that for the present choice of $S_{p e r}$ the bound is off on a $78 \%$ on the real extra value, but if we compare it to

$$
\frac{1}{2 \pi} \int_{-\infty}^{\infty}\left(\left|S_{p e r}\right|-|\hat{S}|\right)^{2} d \omega=0.1366 \omega_{o},
$$

the proposed lower bound differs by $22 \%$. This suggests that for this choice of $S_{p e r}$ and $S_{\max }$, the first approximation of $\left|S_{\text {per }}-\hat{S}\right|^{2}$ by $\left(\left|S_{\text {per }}\right|-|\hat{S}|\right)^{2}$ is weakest, whilst the second approximation performed by replacing $\left(\left|S_{p e r}\right|-|\hat{S}|\right)^{2}$ by $\left(\left|S_{\max }\right|-|\hat{S}|\right)^{2}$ is less coarse. Nonetheless the lower bound obtained through $\left|S_{\max }\right|$ is a more general result since it concludes that for any choice of $S_{p e r}$ that satisfies the conditions exposed in Theorem 4, the extra SNR requirement will be at least of an amount equal to $\omega_{o} / 3 \pi$.

Finally a second choice of $\left|S_{\max }\right|$ has also been investigated

$$
\left|S_{\max }\right|=\left\{\begin{array}{cc}
\varepsilon & 0 \leq \omega \leq \omega_{o}, \\
1 & \omega_{o}<\omega .
\end{array}\right.
$$

The resulting lower bound for this selection is given by $\frac{(1-\varepsilon)^{2}}{\pi} \omega_{o}$.

\section{Conclusion And Remarks.}

In the present paper we have presented the analytical expression for the output feedback sensitivity function achieved when the minimal SNR constraint is imposed. The minimal SNR constraint is such that it is necessary for stabilisability of the closed loop over an ACGN communication channel with bandwidth limitation. The definition of specific control design objectives by means of a target sensitivity function is one useful approach by which the increase in the required SNR (to both account for stabilisability and performance) can be quantified. This result proves to be motivation for the study of the sensitivity reduction as a design requirement. The design requirement is stated by defining frequency bounds on the sensitivity function magnitude. The result of this approach are none tight SNR lower bounds that satisfy the proposed sensitivity reduction.

\section{REFERENCES}

[1] R. Brockett and D. Liberzon. Quantized feedback stabilization of linear systems. IEEE Transactions on Automatic Control, 45(7):12791289, June 2000.

[2] N. Elia and S.K. Mitter. Stabilization of linear systems with limited information. IEEE Transactions on Automatic Control, 46(9):13841400, September 2001.

[3] N. Elia. When Bode meets Shannon: Control-oriented feedback communication schemes. IEEE Transactions on Automatic Control, 49(9):1477-1488, September 2004.

[4] D.E. Quevedo, G.C. Goodwin and J.S. Welsh. Minimizing down-link traffic in networked control systems via optimal control techniques. In Proceedings of the 42nd IEEE Conference on Decision and Control, Maui, USA, December 2003.

[5] S. Tatikonda, A. Sahai and S. Mitter. Control of lqg systems under communication constraints. In Proceedings 37th IEEE Conference on Decision and Control, December 1998.

[6] S. Tatikonda and S.M. Mitter. Control under communication constraints. IEEE Transactions on Automatic Control, 49(7):1056-1068, July 2004.

[7] Special Issue on Networked Control Systems. IEEE Transactions on Automatic Control, 49(9), September 2004.

[8] G.N. Nair and R.J. Evans. Mean square stabilisability of stochastic linear systems with data rate constraints. In Proceedings 41st IEEE Conference on Decision and Control, Las Vegas, USA, December 2002.

[9] G.N. Nair and R.J. Evans. Exponential stabilisability of finitedimensional linear systems with limited data rates. Automatica, 39(4):585-593, April 2003.

[10] G.N. Nair, R.J. Evans, I.M.Y. Mareels and W. Moran. Topological Feedback Entropy for Nonlinear Systems. In Proceedings 5th Asian Control Conference, Melbourne, Australia, July 2004.

[11] N.C. Martins, M.A. Dahleh and J.C. Doyle. Fundamental Limitations of Disturbance Attenuation in the Presence of Side Information (I). In Proceedings of the 44th IEEE Conference on Decision and Control and European Control Conference, Seville, Spain, 2005.

[12] J.H. Braslavsky, R.H. Middleton and J.S. Freudenberg. Feedback stabilisation over signal-to-noise ratio constrained channels. In Proceedings 2004 American Control Conference, Boston, USA, July 2004.

[13] J.H. Braslavsky, R.H. Middleton and J.S. Freudenberg. Feedback Stabilisation over Signal-to-Noise Ratio Constrained Channels. IEEE Transactions on Automatic Control (to appear), 2007.

[14] J.S. Freudenberg, J.H. Braslavsky and R.H. Middleton. Control over Signal-to-Noise Ratio Constrained Channels: Stabilization and Performance. In Proceedings of the 44th IEEE Conference on Decision and Control and European Control Conference, Seville, Spain, December 2005.

[15] J.S. Freudenberg, R.H. Middleton, and V. Solo. The minimal signal-to-noise ratio required to stabilize over a noisy channel. In Proceedings of the 2006 American Control Conference, Minneapolis, USA, June 2006.

[16] A.J. Rojas , J.H. Braslavsky and R.H. Middleton. Output Feedback Control over a class of Signal to Noise Ratio constrained Communication Channels. In Proceedings 2006 American Control Conference, Minneapolis, USA, June 2006.

[17] J.C. Doyle, B.A. Francis and A.R. Tannenbaum. Feedback control theory. Macmillan Publishing Company, 1992.

[18] A.J. Rojas. Feedback Control over Signal to Noise Ratio Constrained Communication Channels. Ph.D. Thesis, The University of Newcastle, University Drive, Callaghan, NSW 2308, Australia, July 2006.

[19] G.H. Goodwin, S.F. Graebe, and M.E. Salgado. Control System Design. Prentice Hall, 2001. 